Ugail H (2007): "Generalized Partial Differential Equations for Interactive Design" International Journal of Shape Modeling, 13 (2): 225-226. 


\title{
Generalized Partial Differential Equations for Interactive Design
}

\author{
Hassan Ugail* \\ School of Informatics \\ University of Bradford \\ Bradford BD7 1DP \\ $U K$ \\ h.ugail@bradford.ac.uk
}

\begin{abstract}
This paper presents a method for interactive design by means of extending the PDE based approach for surface generation. The governing partial differential equation is generalized to arbitrary order allowing complex shapes to be designed as single patch PDE surfaces. Using this technique a designer has the flexibility of creating and manipulating the geometry of shape that satisfying an arbitrary set of boundary conditions. Both the boundary conditions which are defined as curves in 3-space and the spine of the corresponding PDE are utilized as interactive design tools for creating and manipulating geometry intuitively. In order to facilitate interactive design in real time, a compact analytic solution for the chosen arbitrary order PDE is formulated. This solution scheme even in the case of general boundary conditions satisfies exactly the boundary conditions where the resulting surface has an closed form representation allowing real time shape manipulation. In order to enable users to appreciate the powerful shape design and manipulation capability of the method, we present a set of practical examples.
\end{abstract}

Keywords: Interactive Design, Free Form Surfaces, PDEs of arbitrary order

\section{Introduction}

There is no doubt that the search for intuitive and efficient techniques for interactive shape design continues to be a vibrant and demanding area of research. Using established design tools today, designers are increasingly required to produce 3D geometry that mirrors real world objects within shorter time scales.

Broadly speaking interactive design techniques available for a designer today can be put into three categories. They are boundary based methods such as polygon based design ${ }^{18}$, extrusions and surface of revolution ${ }^{25}$, polynomial patches ${ }^{14}$; procedural modeling such as implicit surfaces ${ }^{1}$ and fractals ${ }^{34}$; and volumetric models such as constructive solid geometry ${ }^{30}$ and subdivision ${ }^{11}$. Using these design techniques the common types of geometric operation often carried out include, adding

${ }^{*}$ School of Informatics, University of Bradford, Bradford BD7 1DP, UK. h.ugail@bradford.ac.uk 
detail to various regions of geometry, trimming and blending, Boolean operations and standard geometric transformations. Among the above mentioned techniques the three most widely used are polynomial patches, implicit surfaces and subdivision. Below we discuss each of them briefly.

There exists a wide variety of methods for surface construction using polynomial patches. These include, for example, defining the surface using an array of points, a set of plane cross-sectional curves, or even networks of curves. For instance, earlier work of Tiller ${ }^{36}$ describe techniques using rational B-splines, by which a surface is defined through a series of plane curves . Woodward ${ }^{46}$, on the other hand, describes a wide variety of methods for producing free-form surfaces whereby the surface is defined using a collection of projection and section curves. The most widely used polynomial surfaces for interactive design are the so called NURBS (Non Uniform Rational B-Splines) which offer surface manipulation through manipulation of its control points, weights, and knots ${ }^{27}$. As far as interactive design using spline based techniques are concerned, they often require large arrays of control points which are difficult and time consuming to manipulate. This is especially true when the designer is working in interactive environments. There has been a lot of effort by many researchers to make spline based techniques more intuitive and user friendly. Some of the notable methods proposed include the technique of introducing constraints on the control points ${ }^{16}$, the use of deformations ${ }^{29}$ and the use of Dynamic NURBS or D-NURBS ${ }^{35}$.

When compared to spline based techniques, Implicit functions uses a different metaphor for interactive geometry design whereby functions based on scalar fields are utilized to define geometric entities. Some of the notable shape manipulation techniques that use implicit functions include the use of particles for sampling and controlling the shape ${ }^{45}$, the use of sculpting ${ }^{15,31}$, and morphing ${ }^{37}$.

Subdivision techniques for interactive design have recently emerged to be very popular, where such techniques are seen to be an alternative to traditional splinebased shape modeling. Subdivision techniques are a natural extensions of the existing patch-based surface representations. Subdivision can be regarded as operating directly on polygonal meshes, whereby direct mesh manipulation can be carried out with ease. They can also be used to describe the geometry of objects with arbitrary topology. Furthermore, subdivision based geometry can be modified easily to support features such as creases and boundaries. A wide variety of subdivision schemes for surfaces exist. Two of the most well-known subdivision schemes for surfaces are the Catmull-Clark scheme ${ }^{8}$, and the Loop scheme ${ }^{22}$. For a detailed explanation of subdivision and subdivision surfaces, the interested reader is referred to ${ }^{44}$.

Given the task of interactively creating a complex object on the computer, a designer would go about using one or more of the above techniques. One could argue that non of the above techniques are right neither are they wrong. The particular approach a designer adopts often depends on the type of design and level of familiarity of particular set of techniques. A fine example of this perhaps is the increasing popularity of polygon based design techniques among games developers. 
Surprising though it may be, these designers often prefer to design complex objects and characters polygon by polygon where they have the ultimate control over shape of the model so that they are able to give attention to every minor detail.

In broad terms the existing interactive techniques discussed above could be thought to lie between a spectrum where the extremes are shape design and shape representation. Bearing this in mind, the design techniques discussed in this paper is placed nearer to the shape design than representation.

\subsection{Outline of the PDE Method}

Although from a traditional standpoint PDEs have played a major role in solving engineering related problems such as electromagnetism, stress/strain in physical structures and fluid flows, nowadays PDE based techniques are increasingly becoming very popular in many application areas of geometric design. These include level set formulations for image processing, computer graphics and computer vision $^{32,26,43}$, mesh fairing ${ }^{33,10}$, Radial Basis Functions ${ }^{28}$, biharmonic Bézier surfaces $^{24}$ and polysplines ${ }^{21}$.

The work discussed in this paper is based around the pioneering work of Bloor and Wilson on the so called PDE method ${ }^{2}$. The particular approach we use adopts a boundary-value approach whereby a surface is characterized by defining a number of space curves (with associated derivative information) so as to form the surface's edges, and then the surface is generated between these curves by solving an elliptic partial differential equation (PDE). The chosen PDE is usually lower order such as the Biharmonic equation.

The PDE method for geometric design was originally developed as a mechanism of blend shape generation. Since this initial application of the method on shape blending, in recent years PDE based shapes have broadened their uses in shape description ${ }^{20,12,38}$. This is due to the fact that PDE based shapes in principle have the advantage that most of the information defining a shape is based on a boundary representation making it possible for complex geometry to be generated and controlled by very few design parameters ${ }^{40}$.

The method has been successfully utilized for automatic design for function in various design scenarios by means of incorporating engineering design criteria such as functional constraints into the geometric design of PDE surfaces. Examples of this include automatic design of ship hulls ${ }^{23}$, propeller blades ${ }^{9}$, engineering components ${ }^{4}$ and thin-walled structures ${ }^{39}$. Thus, it has been shown that by utilizing the PDE method it is possible to introduce geometric constraints, aesthetic criteria, and physical and engineering restrictions into the design process. Moreover, numerical algorithms have also been developed to approximate PDE surfaces using standard B-splines ${ }^{3,19}$. This work demonstrates that PDE surface are compatible with existing data exchange standards and therefore can be readily incorporated into existing commercial design systems.

On the interactive design front there has been a number of developments which 
centre around the use of low order PDEs. For the example, Du and Qin have developed interactive tools for sculpting PDE shapes by incorporating physicsbased modeling techniques ${ }^{13,12}$. They also proposed a number of new techniques for local control of shapes generated using the PDE method. To demonstrate their techniques they have developed an interactive toolkit that utilises the PDE method to model the real-world objects with interior material distribution. Furthermore, their recent work also includes the integration of implicit functions with PDEs in order to demonstrate that solid modeling based on the PDEs can potentially unify both geometric constraints and functional requirements within a single design framework ${ }^{13}$. The work of Zhang and colleagues on interactive design and efficient solution formulation for PDE based surfaces is also highly notable here ${ }^{49,47}$.

This paper presents a methodology by which the original Biharmonic based PDE model of Bloor and Wilson can be generalized for interactive design. Thus, we show how the governing partial differential equation is generalized to arbitrary order allowing complex shapes to be designed as single patch PDE surfaces. Using this technique a designer has the flexibility of creating and manipulating the geometry of a shape that satisfy an arbitrary set of boundary conditions. Both the boundary conditions which are defined as curves in 3-space and the spine of the corresponding $\mathrm{PDE}$ are utilized as interactive design tools for creating and manipulating geometry interactively.

Thus, from the point of view of shape modeling in an interactive environment, the generalization of PDE surfaces to arbitrary order presents a number of added advantages. For example, in the case of generating a complex surface patch, using low order PDE surfaces, it is often necessary to use a collection of PDE patches to smoothly interpolate between a series of curves, where manual intervention of trimming and stitching is often necessary. This is especially the case where the derivative boundary curves have to be manually adjusted in order to ensure continuity and smoothness across blend surfaces ${ }^{38}$. It would be highly desirable to have mechanisms in place in order to reduce the number of PDE patches that can be used to describe a complicated shape. In fact in certain circumstances it would be ideal to have a single PDE patch, which would allow the production of a smooth surface patch through a collection of curves where the number of curves would determine the order of the chosen PDE. Other advantages of using higher order PDEs include the ability to efficiently parameterize a complex surface so that in principle the user can be presented with fewer number of meaningful shape parameters through which a user can undertake interactive design with ease.

The paper is organized as follows. Section (2) presents the generalized framework and presents an analytic solution that can be utilized to create geometry in real time. The discussions in Section (3) focus on how the various parameters associated with the chosen PDE can be appropriately chosen so as smooth surfaces can be generated. A set of examples to demonstrate the capability of the method to create complex geometry is also given. In Section (4) we discuss interactive design using higher order PDEs. The discussion on this section focuses on how the boundary 
conditions can be chosen and utilized to manipulate geometry interactively in real time. Furthermore, the generalization of a powerful interactive tool known as the spine of the PDE surface is also discussed.

\section{Generalized PDEs}

We assume that a parametric surface patch is defined by a function $\mathbf{X}(u, v)$ such that,

$$
\mathbf{X}(u, v)=(x(u, v), y(u, v), z(u, v))
$$

where $u$ and $v$ are parameters for a finite two-dimensional region $\Omega$ such that $\Omega$ is the region $\{u, v: 0 \leq u \leq 1 ; 0 \leq v \leq 2 \pi\}$ which map onto a point in physical space $(x, y, z)$. In the present formulation we require an elliptic PDE that satisfies a given number of $2 N$ boundary conditions. Here $N$ is an arbitrary integer such that $N \geq 2$. The boundary conditions can be written in the form,

$$
\begin{aligned}
\mathbf{X}(0, v) & =\mathbf{f}_{1}(v), \\
\mathbf{X}\left(u_{i}, v\right) & =\mathbf{g}_{i}(v), i=2 \ldots 2 N-1 \\
\mathbf{X}(1, v) & =\mathbf{f}_{2 N}(v),
\end{aligned}
$$

where $\mathbf{f}_{1}(v)$ in Equation (2) and $\mathbf{f}_{2 N}(v)$ in Equation (4) are function boundary conditions specified at $u=0$ and $u=1$ respectively. The conditions $\mathbf{X}\left(u_{i}, v\right)=$ $\mathbf{g}_{i}(v)$ in Equation (3) can take the form either

$$
\mathbf{X}\left(u_{i}, v\right)=\mathbf{f}_{i}(v) \text { for } 0<u_{i}<1, i=2 \ldots 2 N-1,
$$

or

$$
\frac{\partial \mathbf{X}}{\partial u}, \frac{\partial^{2} \mathbf{X}}{\partial u^{2}}, \frac{\partial^{3} \mathbf{X}}{\partial u^{3}}, \ldots, \frac{\partial^{2 N-2} \mathbf{X}}{\partial u^{2 N-2}} \text { for } 0 \leq u_{i} \leq 1, i=2 \ldots 2 N-1 .
$$

In simpler terms the above boundary condition implies that for a PDE surface patch of order $2 N$ we can specify two function boundary conditions, as given in Equations (2) and (4), that should be satisfied at the edges (at $u=0$ and $u=1$ ) of the surface patch, and a number of function or derivative conditions, as given in Equation (3), amounting to $2 N-2$ boundary conditions which the PDE should also satisfy.

With the above formulation we take standard Laplace operator, $\nabla=0$, as a base PDE and generalize it to the $N^{t h}$ order such that,

$$
\left(\frac{\partial^{2}}{\partial u^{2}}+a^{2} \frac{\partial^{2}}{\partial v^{2}}\right)^{N} \underline{X}(u, v)=0
$$

As one can easily observe the above equation is a generalization of the usual 4th order elliptic PDE where the corresponding Biharmonic equation can be derived by choosing $N$ to be 2 . 


\subsection{Analytic Solution of Generalized PDEs}

Given a set of $2 N$ boundary conditions as defined in Equations (2), (3) and (4), we take the $(u, v)$ parameter space $\Omega$ to be the region $\{u, v: 0 \leq u \leq 1 ; 0 \leq v \leq 2 \pi\}$. Thus, we assume that all the boundary conditions are periodic in $v$ in the sense $\mathbf{f}_{0}(0)=\mathbf{f}_{0}(2 \pi), \mathbf{f}_{1}(0)=\mathbf{f}_{1}(2 \pi)$ and $\mathbf{g}_{i}(0)=\mathbf{g}_{i}(2 \pi)$. We further assume that all the boundary conditions are continuous functions within the domain of $\Omega$.

With the above assumptions on the boundary conditions we can utilize the method of separation of variables to write the analytic solution of Equation (7) as,

$$
\mathbf{X}(u, v)=\mathbf{A}_{0}(u)+\sum_{n=1}^{\infty}\left[\mathbf{A}_{n}(u) \cos (n v)+\mathbf{B}_{n}(u) \sin (n v)\right],
$$

where

$$
\mathbf{A}_{0}=\mathbf{a}_{00}+\mathbf{a}_{01} u+\mathbf{a}_{02} u^{2}+\ldots+\mathbf{a}_{(2 N-1)} u^{2 N-1},
$$

$$
\begin{aligned}
& \mathbf{A}_{n}=\mathbf{a}_{n 1} e^{a n u}+\mathbf{a}_{n 2} u e^{a n u}+\mathbf{a}_{n 3} e^{-a n u}+\mathbf{a}_{n 4} u e^{-a n u}+\ldots+ \\
& \mathbf{a}_{n(2 N-3)} u^{N-2} e^{a n u}+\mathbf{a}_{n(2 N-2)} u^{N-1} e^{a n u}+\mathbf{a}_{n(2 N-1)} u^{N-2} e^{-a n u}+ \\
& \mathbf{a}_{n 2 N} u^{N-1} e^{-a n u},
\end{aligned}
$$

$$
\begin{aligned}
& \mathbf{B}_{n}=\mathbf{b}_{n 1} e^{a n u}+\mathbf{b}_{n 2} u e^{a n u}+\mathbf{b}_{n 3} e^{-a n u}+\mathbf{b}_{n 4} u e^{-a n u}+\ldots+ \\
& \mathbf{b}_{n(2 N-3)} u^{N-2} e^{a n u}+\mathbf{b}_{n(2 N-2)} u^{N-1} e^{a n u}+\mathbf{b}_{n(2 N-1)} u^{N-2} e^{-a n u}+ \\
& \mathbf{b}_{n 2 N} u^{N-1} e^{-a n u},
\end{aligned}
$$

where $\mathbf{a}_{00}+\mathbf{a}_{01}, \mathbf{a}_{02}, \ldots, \mathbf{a}_{2 N-1}, \mathbf{a}_{n 1}+\mathbf{a}_{n 2}, \mathbf{a}_{n 3}, \mathbf{a}_{n 4}, \ldots, \mathbf{a}_{n(2 N-3)}, \mathbf{a}_{n(2 N-2)}$, $\mathbf{a}_{n(2 N-1)}, \mathbf{a}_{n 2 N}$ and $\mathbf{b}_{n 1}+\mathbf{b}_{n 2}, \mathbf{b}_{n 3}, \mathbf{b}_{n 4}, \ldots, \mathbf{b}_{n(2 N-3)}, \mathbf{b}_{n(2 N-2)}, \mathbf{b}_{n(2 N-1)}, \mathbf{b}_{n 2 N}$ are vector-valued constants, whose values are determined by the imposed boundary conditions at $u_{i}$ where $0 \leq u_{i} \leq 1$.

Since the chosen boundary conditions are all continuous functions which are also periodic in $v$ we can write down their Fourier series representation as,

$$
\begin{aligned}
\mathbf{f}_{1}(v) & =\mathbf{C}_{0}^{1}+\sum_{n=1}^{\infty}\left[\mathbf{C}_{n}^{1} \cos (n v)+\mathbf{S}_{n}^{1} \sin (n v)\right] \\
\mathbf{g}_{i}(v) & =\mathbf{C}_{0}^{i}+\sum_{n=1}^{\infty}\left[\mathbf{C}_{n}^{i} \cos (n v)+\mathbf{S}_{n}^{i} \sin (n v)\right], i=2 \ldots 2 N-1, \\
\mathbf{f}_{2 N}(v) & =\mathbf{C}_{0}^{2 N}+\sum_{n=1}^{\infty}\left[\mathbf{C}_{n}^{2 N} \cos (n v)+\mathbf{S}_{n}^{2 N} \sin (n v)\right] .
\end{aligned}
$$

Let us assume for the moment that the Fourier sums in the Expressions (12), (13) and (14) has finite $M$ modes. Then the vector constants $\mathbf{C}_{0}^{1}, \mathbf{C}_{0}^{i}$ for $i=2 \ldots 2 N-1$, and $\mathbf{C}_{0}^{2 N}$ can be obtained by directly comparing them with the constants $\mathbf{a}_{00}$, 
$\mathbf{a}_{01}, \mathbf{a}_{02}, \ldots, \mathbf{a}_{(2 N-1)}$ given in Equation (9). Now for each of the Fourier modes $n=\{1, \ldots, M\}$ we can write linear systems,

$$
\left(\begin{array}{c}
\mathbf{a}_{n 1} \\
\vdots \\
\mathbf{a}_{2 N}
\end{array}\right)=\mathbf{A}(a, n)\left(\begin{array}{c}
\mathbf{C}_{n}^{1} \\
\vdots \\
\mathbf{C}_{n}^{2 N}
\end{array}\right)
$$

and

$$
\left(\begin{array}{c}
\mathbf{b}_{n 1} \\
\vdots \\
\mathbf{b}_{2 N}
\end{array}\right)=\mathbf{B}(a, n)\left(\begin{array}{c}
\mathbf{S}_{n}^{1} \\
\vdots \\
\mathbf{S}_{n}^{2 N}
\end{array}\right)
$$

where $\mathbf{A}(a, n)$ and $\mathbf{B}(a, n)$ are $2 N \times 2 N$ matrices, whose coefficients can be obtained by solving the linear systems (15) and (16) subject to the Fourier coefficients corresponding to the $2 N$ boundary conditions.

The above solution scheme is based on the fact that the boundary conditions can be expressed as a finite Fourier series. However, in practical design scenarios such an assumption is not a realistic proposition. In order to take care of this, we adopt a generalized version of the spectral approximation to the Biharmonic PDE given in ${ }^{6}$ as described below.

Although in practical terms we cannot assume that a given boundary condition can be expressed accurately using a finite Fourier series, it is reasonable to assume that the boundary conditions can be written as,

$$
\begin{aligned}
\mathbf{f}_{1}(v) & =\mathbf{C}_{0}^{1}+\sum_{n=1}^{M}\left[\mathbf{C}_{n}^{1} \cos (n v)+\mathbf{S}_{n}^{1} \sin (n v)\right]+\mathbf{R}_{1}(v), \\
\mathbf{g}_{i}(v) & =\mathbf{C}_{0}^{i}+\sum_{n=1}^{M}\left[\mathbf{C}_{n}^{i} \cos (n v)+\mathbf{S}_{n}^{i} \sin (n v)\right]+\mathbf{R}_{i}(v), i=2 \ldots 2 N-1, \\
\mathbf{f}_{2 N}(v) & =\mathbf{C}_{0}^{2 N}+\sum_{n=1}^{M}\left[\mathbf{C}_{n}^{2 N} \cos (n v)+\mathbf{S}_{n}^{2 N} \sin (n v)\right]+\mathbf{R}_{2 N}(v) .
\end{aligned}
$$

Thus, the basic idea here is to formulate each of the boundary conditions in terms of the sum of a finite Fourier series containing $M$ modes and a 'remainder' term $\mathbf{R}_{i}(v), i=1 \ldots 2 N$ which contains the higher order Fourier modes. In Ref. 6, for the case of Biharmonic equation, it is shown that the higher order Fourier modes make negligible contributions to the interior of the PDE patch and the same applies for the general case of $N^{t h}$ order Biharmonic PDE. Hence it is reasonable to truncate the Fourier series at some finite $M$, (typically $M=6$ is adequate), and represent the contribution of the high frequency modes to the surface with a remainder function $\mathbf{R}(u, v)$. The format of this remainder function is somewhat arbitrary and for this 
work it is taken to be of the form,

$$
\begin{aligned}
& \mathbf{R}(u, v)=\mathbf{r}_{1} e^{\omega u}+\mathbf{r}_{2} u e^{\omega u}+\mathbf{r}_{3} e^{-\omega u}+\mathbf{r}_{4} u e^{-\omega u}+\ldots+ \\
& \mathbf{r}_{(2 N-3)} u^{N-2} e^{\omega u}+\mathbf{r}_{(2 N-2)} u^{N-1} e^{\omega u}+\mathbf{r}_{(2 N-1)} u^{N-2} e^{-\omega u}+ \\
& \mathbf{r}_{2 N} u^{N-1} e^{-\omega u}
\end{aligned}
$$

where $\mathbf{r}_{1}, \mathbf{r}_{2} \ldots \mathbf{r}_{(2 N-1)}, \mathbf{r}_{2 N}$ are vector-valued constants which depend on $v$.

Now by taking $\widetilde{\mathbf{X}}(u, v)$ to the approximate solution,

$$
\widetilde{\mathbf{X}}(u, v)=\mathbf{A}_{0}(u)+\sum_{n=1}^{M}\left[\mathbf{A}_{n}(u) \cos (n v)+\mathbf{B}_{n}(u) \sin (n v)\right],
$$

satisfying the boundary conditons of the finite Fourier series we define difference functions such that,

$$
\begin{aligned}
\mathbf{d f}_{1}(v) & =\mathbf{d f}_{1}(v)-\widetilde{\mathbf{X}}(0, v), \\
\mathbf{d g}_{i}(v) & =\mathbf{g}_{i}(v)-\widetilde{\mathbf{X}}\left(u_{i}, v\right), i=2 \ldots 2 N-1, \\
\mathbf{d f}_{2 N}(v) & =\mathbf{d f}_{1}(v)-\widetilde{\mathbf{X}}(1, v)
\end{aligned}
$$

By choosing $\omega$ in the Expression (20) to be an, the vector constants $\mathbf{r}_{1} \ldots \mathbf{r}_{2 N}$ can be computed by means of direct comparison with the diffrence terms $\mathbf{d f}_{1}(v)$, $\mathbf{d f}_{2 N}(v)$ and $\mathbf{d g}_{i}(v)$, for $i=2 \ldots 2 N-1$ in Equations (22) - (24).

Finally the approximate analytic solution of the PDE is given as,

$$
\mathbf{X}(u, v)=\tilde{\mathbf{X}}(u, v)+\mathbf{R}(u, v) .
$$

It is important to note that the choice of the number of Fourier terms $M$ will affect how well $\widetilde{\mathbf{X}}(u, v)$ approximates the solutions of the general $N^{t h}$ order Biharmonic PDE. Although this may be the case, due to the choice of difference functions utilized here, i.e. by computing the difference between the original boundary conditions and the corresponding finite Fourier series, as described in Equations (22) (24) the approximate solution satisfies the chosen set of boundary conditions exactly (to within the machine accuracy).

The main aim here is to obtain solutions for the $N^{t h}$ order Biharmonic PDE surfaces with general boundary conditions rapidly enough to be able carry out interactive design in real-time. The above solution method can be viewed as a spectral method for the solutions of arbitrary order linear elliptic PDEs where the expansion functions are based upon the Fourier series. Thus, this solution scheme can be applied in the case of general complex periodic boundary conditions which can be seen to be suitable enough for generating a wide range of complex geometries. In the case of non-periodic boundary conditions, an analytic approximation for the Biharmonic case has been discussed in Ref. 7 which in principle can also be generalized to suit for higher order PDEs. 


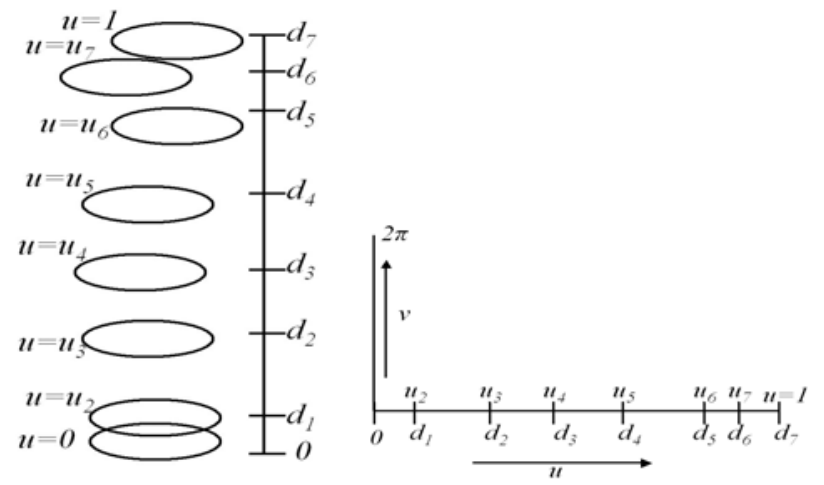

(a)

(b)

Fig. 1. Choice of ' $u$ ' parameterization for higher order PDEs.

\section{PDE Geometries of Generalised Higher Order Equations}

For reasons that will become clearer later, for the purpose of interactive design and manipulation of surfaces using higher order PDEs intuitively it is desirable to choose low order derivative boundary conditions, for example derivative condition of up to order 4 . This, therefore, requires us to choose a number of function boundary conditions where the surface patch will then pass through these boundary conditions satisfying them exactly. Thus, in a sense we could envisage interactive design using higher order PDEs being able to define an arbitrary set of boundary curves in the physical space where the surface patch interpolates through most of these curves.

Bearing the above fact in mind here we provide a recipe for the choice of the parameter $a$ and $u$ parameterization in order that a smooth surface is generated as a solution to an arbitrary order $N^{t h}$ order Biharmonic PDE. Similar to previous work on interactive design ${ }^{38}$ here we assume that for the purpose of interactive design a user generates and manipulates a series of general curves in the physical space which enable to define the corresponding boundary conditions for the PDE.

\subsubsection{Choice of ' $u$ ' parameterization}

The main point to bear in mind when choosing the parameterization in the $u$ direction is that one should allow for the possibility of non-linear variation in the Euclidean distances between individual curves defining the PDE boundary conditions.

Figure 1(a) shows a typical set of 8 boundary curves in 3-space which may be utilized to define the boundary conditions for the $8^{t h}$ order PDE. As can be noted the physical variations between these curves are not necessarily linear. This should be taken into account when the corresponding $u$ parameterization is constructed on the $(u, v)$ parameter space. In order to do this the following simple procedure is adopted. First the average Euclidean distance between individual curves are deter- 
mined. Then, the distribution of $u_{i}$ along $u$ parameter direction is proportionally determined where the $u=0$ takes the position for the first boundary curve and $u=1$ takes the position for the last boundary curve. This procedure for constructing $u$ parameterization is illustrated in Figure 1(b).

\subsubsection{Choice of ' $a$ ' parameter}

As noted earlier for reason of intuitively in interactive design using higher PDEs, generally we choose boundary curves such that most of them are function boundary condition rather than higher order derivative boundary conditions. For higher order PDEs the parameter $a$ therefore has a different meaning when compared to interactive design using lower order PDEs such as the Biharmonic case where two function boundary conditions and two derivative boundary conditions are chosen at the edges of the surface patch. Thus, in the case of Biharmonic equation the interior surface patch can solely be controlled from these boundary conditions defined at the edges of the surface patch.

In the case of the Biharmonic PDE the parameter $a$ determines the rate at which the corresponding PDE solution 'forgets' about the boundary condition as one moves away from a boundary into the surface interior. This fact is utilized in Ref. 38 to allow $a$ to be an interactive design parameter where $a$ controls the 'waist' of the surface patch, in that the higher the value of $a$ the more prominent the waist is.

As one can clearly see that for higher order PDEs where the surface is required to pass through a set of intermediate curves (apart from the two edge curves), and in such a scenario the parameter $a$ cannot be utilized to bring about a waisting effect to the interior of the surface patch. In fact, looking at the solution of the $N^{t h}$ order Biharmonic PDE given in Equation (8) we can see that we are asking for an infinitely differentiable function which should pass through a series of space curves whose variation may not necessarily be linear in the physical space. Thus, similar to the problem of data interpolation using spline functions ${ }^{17}$ it is possible for a PDE surface to posses ripples within the interior of the patch. However a careful choice of $a$ to suit a given number of boundary conditions takes care of this problem. To do this we make the following observations on the behavior of $a$ on the differential operator given in Equation (7).

From the exponential terms involving $a$, in the Equations (10) and (11) we note that the value of $a$ influences the decay of the amplitude of the Fourier coefficients through the exponential terms and therefore has an influence on the cross sectional shape of the surface patch. In fact decreasing the value of $a$ has a decreasing effect on the smoothness of the cross sectional shape of the surface patch. In the limit when $a \rightarrow 0$ the $N^{t h}$ order biharmonic PDE becomes a $2 N^{t h}$ order ordinary differential equation whose solution is a Hermite surface.

From the above discussions it is clear that if we are to generate a higher order PDE surfaces, where a large number of position boundary conditions are specified, 


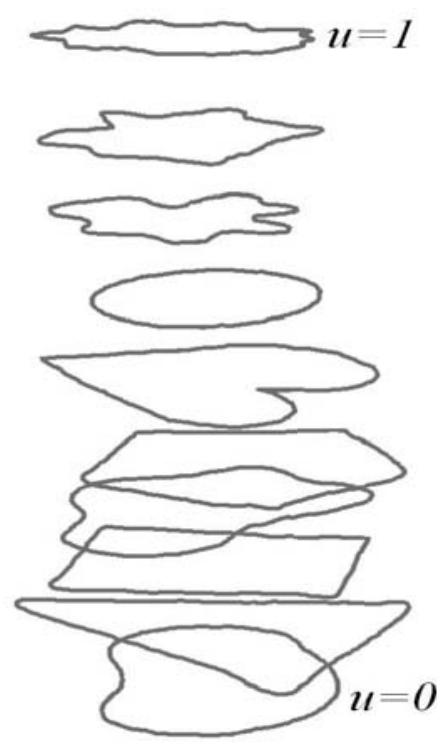

(a)

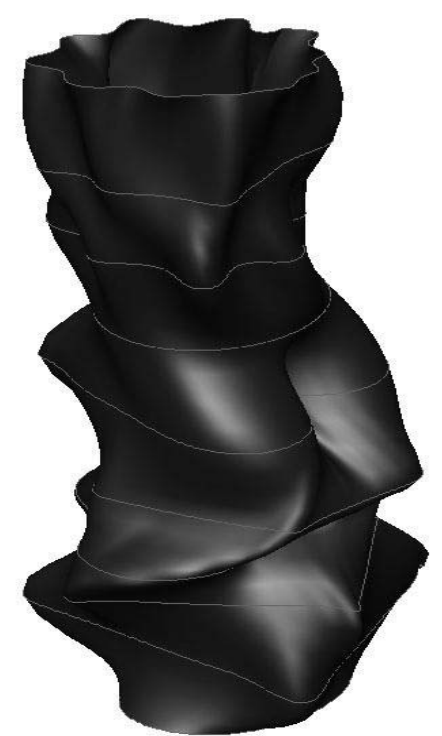

(b)

Fig. 2. A typical higher order PDE patch generated as a solution to the $10^{\text {th }}$ order equation.

we should take a suitable higher value for $a$ depending on the number of position boundary conditions so as the cross-sectional smoothness of the surface patch is maintained. For the work described here for a PDE involving $2 N$ position boundary conditions we have chosen $a$ to be $N$.

\subsection{Example Geometries}

In order to demonstrate the capability of complex surface generation using higher order equations we discuss several examples. These examples are constructed by means of defining a series of cross-sectional curves in 3-space where such curves forms the profile geometry. Thus, in all cases the curves were taken to be function boundary curves through which the surface is required pass. For each case the corresponding PDE is solved within an interactive environment.

Figure (2)(b) shows a higher order PDE surface patch of order 10. The corresponding position boundary curves are shown in Figure (2)(a) where these curves are all free-hand curves generated as cubic B-splines within an interactive graphical environment. The surface is then generated and rendered in real time where the corresponding curves are also shown on the surface.

Figure (3) shows the shape of a sea shell generated as a single patch PDE surface 


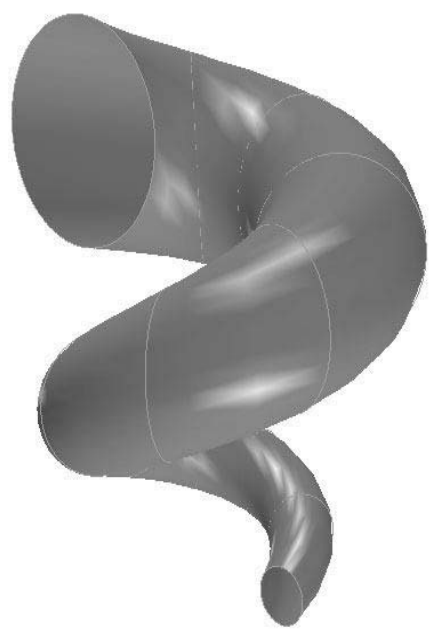

Fig. 3. The shape of a sea shell generated as a PDE patch of order 12

of order 12. The boundary conditions for this surface is taken to be,

$$
\begin{array}{r}
x=\alpha(1-u / 2 \pi) \cos (2 u)(1+\cos (v))+\gamma \cos (2 u), \\
y=\beta(1-u /(2 \pi)) \sin (2 u)(1+\cos (v))+\gamma \sin (2 u), \\
z=\gamma u / 2 \pi+\alpha(1-v /(2 \pi)) \sin (v) .
\end{array}
$$

where 12 cross sectional ellipses along $u$ were taken with $0 \leq u \leq \pi, 0 \leq v \leq 2 \pi$, $\alpha=0.2, \beta=1.0$ and $\gamma=0.1$.

Figure 4 shows the familiar shape of the Klein bottle generated as solution to the $40^{\text {th }}$ order PDE. Again similar to the previous case of sea shell shape the cross section curves of the Klein bottle was taken to be analytic functions as given below.

$$
\begin{aligned}
& x= \begin{cases}\alpha \cos (u)(1+\sin (u))+\gamma \cos (u) \cos (v) & 0 \leq u<\pi \\
\alpha \cos (u)(1+\sin (u))+\gamma \cos (v+\pi) & \pi \leq u \leq 2 \pi\end{cases} \\
& y= \begin{cases}\beta \sin (u)+\gamma \sin (u) \cos (v) & 0 \leq u<\pi \\
\beta \sin (u) & \pi \leq u \leq 2 \pi\end{cases} \\
& z=\gamma \sin (v)
\end{aligned}
$$

where 40 cross sectional ellipses along $u$ were taken with $0 \leq v \leq 2 \pi, \alpha=6.0$, $\beta=16.0$ and $\gamma=4(1-\cos (u) / 2)$.

In order to test the real time capability of surface generation using the proposed method, for each of the examples mentioned above the time it took to generate the surface was computed. These computations were performed on a Laptop computer with a $2.8 \mathrm{GHz}$ processor in which an interactive environment was set up using Microsoft Visual $\mathrm{C}++$ coupled with OpenGL. Note although analytic representations of the boundary conditions were available for both the sea shell and Klein bottle, 


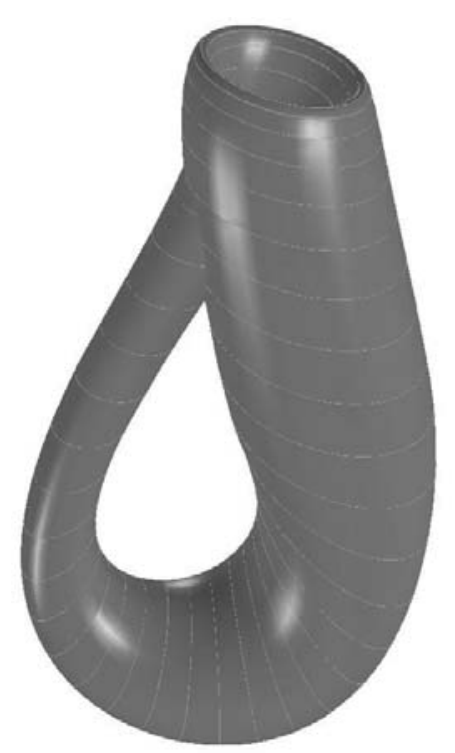

Fig. 4. The Klein bottle PDE surface generated as solution to $40^{\text {th }}$ order PDE

\begin{tabular}{c|ccc} 
& $u$ & $v$ & time (milliseconds) \\
\hline Figure (2) & 20 & 30 & 61 \\
Figure (3) & 30 & 10 & 94 \\
Figure (4) & 40 & 10 & 238
\end{tabular}

Table 1. Computation time along with the subdivisions taken along the parameter directions.

in all cases a discrete set of 300 points along each of the curves were taken where a separate routine was used to generate Fourier representations of the curves with 6 Fourier modes. Table 1 shows the time it took to generate the geometries where the number of subdivisions taken along the parameter directions are also tabulated.

\section{Interactive Design using Higher Order PDEs}

It is clear that for interactive design the most intuitive framework for defining the various PDE boundary conditions is through a series of curves in 3-space where such curves can be manipulated within a graphical environment so that the shape of the surface can be foreseen by the designer. Thus, for the case of Biharmonic equation, in previous works on interactive design, the boundary conditions (both in the case of functions and derivatives) are defined to be 4 curves in 3-space where two of them corresponds to the edges of the surface patch while the other two defines the direction at which the surface patch leaves the boundary. One should also note that in the case of Biharmonic equation the boundary conditions are solely defined 


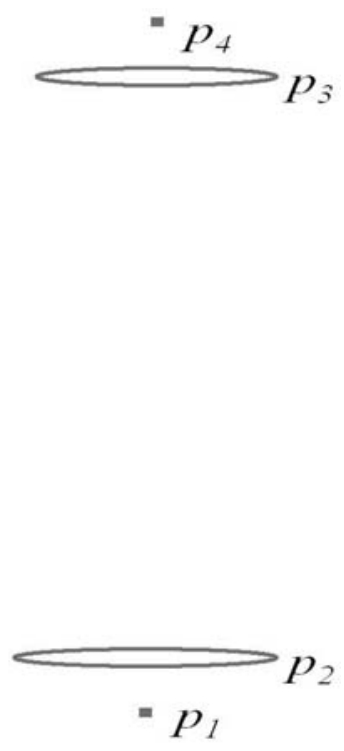

(a)

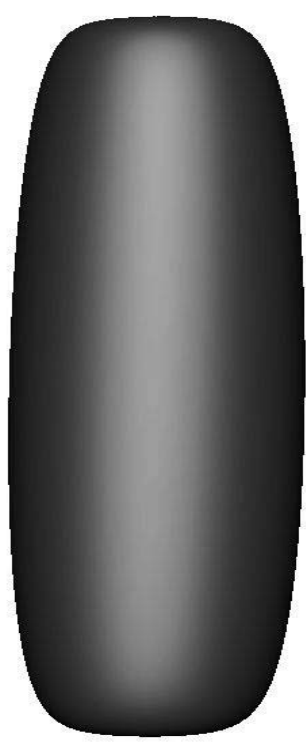

(b)

Fig. 5. A basic membrane shape designed as solution to the biharmonic equation with position boundary curves. (a) The position boundary curves (b) The corresponding surface

at the edges (at $u=0$ and $u=1$ ) of the surface patch through which the interior shape of the surface can be controlled.

For higher order PDEs one could, in principle, utilize the same framework where function boundary conditions and a number of derivative boundary conditions can be defined at the two edges of the surface patch. However, as discussed below, for interactive design purposes choosing too many derivative boundary conditions near the edge of the surface patch does not present an intuitive interface since an excessive number of space curves can be seen to be cluttered near the boundary of the surface patch.

Therefore, as far as possible one should try and distribute the boundary conditions throughout the surface patch in order to enable intuitive surface manipulation. Moreover, apart from the boundary curve manipulation, the spine of the PDE surface ${ }^{41,42}$ can also be utilized as an added design tool. Detailed discussions of how the spine of higher order PDE surfaces can be used to manipulate surface is also described below.

\subsection{Choosing Boundary Conditions for Interactive Design}

In order to provide an understanding of how the boundary curves can be chosen for interactive design using higher order PDEs, here we demonstrate the design of a practical object namely the design and manipulation of a fluid membrane.

Figure (5)(b) shows the basic fluid membrane shape such as that corresponding 
to a biological vesicle. The shape is generated as a solution to the usual biharmonic equation where only function boundary conditions were used so that the geometry of the shape contains the corresponding boundary curves shown Figure (5)(a)

To introduce complexity within the interior of this membrane shape we add a set of derivative boundary conditions which are also specified as curves in the physical space. The order of the PDE is then increased appropriately and the new shape is generated as shown in Figure (6)(b). The added boundary conditions are taken to be the first and second derivative conditions at the $u$ positions corresponding to the position curves $p_{2}$ and $p_{3}$ such that,

$$
\begin{aligned}
& \left.\frac{\partial \mathbf{X}}{\partial u}\right|_{\mathbf{p}_{\mathbf{2}}}=\left[\mathbf{d}_{\mathbf{1}}(v)-\mathbf{p}_{\mathbf{2}}(v)\right] \alpha, \\
& \left.\frac{\partial \mathbf{X}}{\partial u}\right|_{\mathbf{p}_{\mathbf{3}}}=\left[\mathbf{p}_{\mathbf{3}}(v)-\mathbf{d}_{\mathbf{2}}(v)\right] \beta,
\end{aligned}
$$

and

$$
\begin{aligned}
& \left.\frac{\partial^{2} \mathbf{X}}{\partial u^{2}}\right|_{\mathbf{p}_{\mathbf{2}}}=\left[\mathbf{p}_{\mathbf{2}}(v)-2 \mathbf{d}_{\mathbf{1}}(v)+\mathbf{t}_{\mathbf{1}}(v)\right] \gamma, \\
& \left.\frac{\partial^{2} \mathbf{X}}{\partial u^{2}}\right|_{\mathbf{p}_{3}}=\left[\mathbf{p}_{\mathbf{3}}(v)-2 \mathbf{d}_{\mathbf{2}}(v)+\mathbf{t}_{\mathbf{2}}(v)\right] \eta,
\end{aligned}
$$

where $\alpha, \beta, \gamma$ and $\eta$ are constants.

As the format of the Equations (32) - (35) suggests, the definitions of the deriavative boundary conditons resemble that of a finite-difference approximation scheme. As mentioned previously, it is important to note that the surface patch does not necessarily pass through the curves that define the derivative boundary conditions.

To gain an understanding of how the boundary conditions, in particular the derivative conditions, affect the geometry of a higher order surface, consider the following simplification. Following the discussion of the effect of $a$ on the surface patch in Section (3.0.2) we note that in the limit when $a \rightarrow 0$ the corresponding PDE surface becomes a Hermite surface such that the curves of constant $v$ are Hermite curves. Although due to the smoothing effect of the PDE $(a \neq 0)$ the curves of constant $v$ are not Hermite, but considering them in these terms is useful in this present situation. Thus, drawing this similarity, for lines of constant $v$ we can think that there exist $(2 N-1)^{t h}$ order polynomial function. Such a polynomial curve can be seen as being defined by a series of control points which control the position and derivatives of the curve along various points. In the case of the membrane shape shown in Figure (6)(b) we can imagine four control points that control the edges and positions at $p_{2}$ and $p_{3}$, two control points controlling the tangent and a further two control points controlling the curvatures. Thus, the membrane shape can be seen to be controlled by eight continuous curves in space.

From Figure (6)(b) one could make another important observation regarding the effect of the derivative conditions on the surface patch. That is, the regions of the surface affected by the derivative curves are within close proximity of the corresponding position curves. In fact what one would observe here is, at a particular 


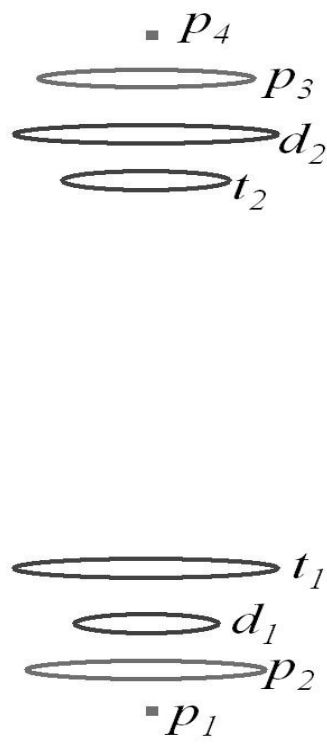

(a)

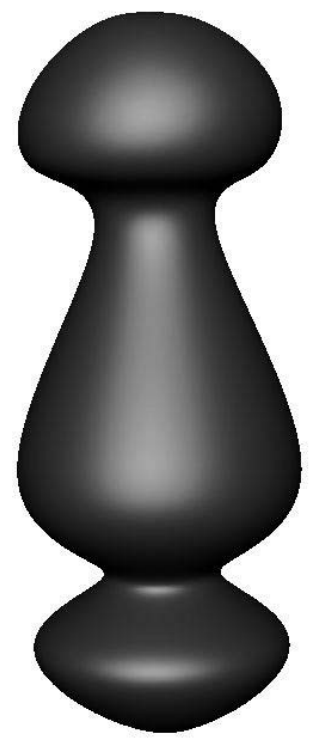

(b)

Fig. 6. A complex membrane shape designed as soluton to higher order PDE with a number of position and derivative boundary conditions. (a) Position and derivative boundary curves (b) The corresponding surfac patch.

point on the surface, as the order of derivative conditions increases the effect of them on the surface patch is drawn closer and closer to that point on the surface. Furthermore, as noted earlier the surface patch does not necessarily pass through derivative curves. Thus, as the order of derivative conditions are increased at a particular position on the surface, the surface manipulation using such curves becomes non-intuitive as the scenario will then have a similar meaning to a spline surface manipulation using a set of closely related control points.

\subsection{Spine of Higher Order PDEs}

If we now draw our attention to the term $\mathbf{A}_{0}$ in the Equation (9), we note that it takes the form of a polynomial of degree $(2 N-1)$. By looking at the structure of the PDE solutions given in Equation (8) we can arrive to the following conclusions.

From the structure of solution to $2 N^{t h}$ order PDE given in Equation (8) we observe that a surface point $\mathbf{X}(u, v)$ can be thought of being composed of sum of a vector $\mathbf{A}_{0}$ giving the 'center line' of the surface and a radius vector defined by the term $\sum_{n=1}^{\infty}\left[\mathbf{A}_{n}(u) \cos (n v)+\mathbf{B}_{n}(u) \sin (n v)\right]$ providing the position of $\mathbf{X}(u, v)$ relative to the "center line. Thus, the polynomial in the $\mathbf{A}_{0}$ term describes the spine or the skeleton of the PDE surface. Detailed discussions on the spine of the PDE for the Biharmonic case is provided $\mathrm{in}^{41,42}$ where it has been shown that the spine of a PDE surface can also be utilized as a powerful as well as an intuitive shape 


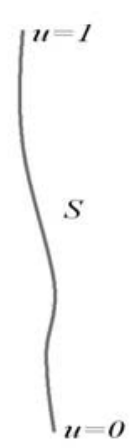

(a)

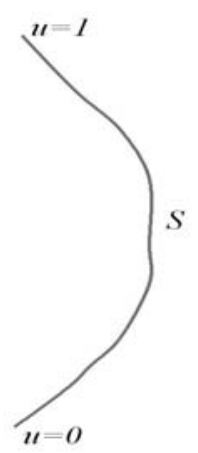

(b)

Fig. 7. Spine of Higher order PDEs. (a) The spine of the typical higher order PDE surface shown in Figure (2) (b) The spine of the manipulated surface shown in Figure (8)

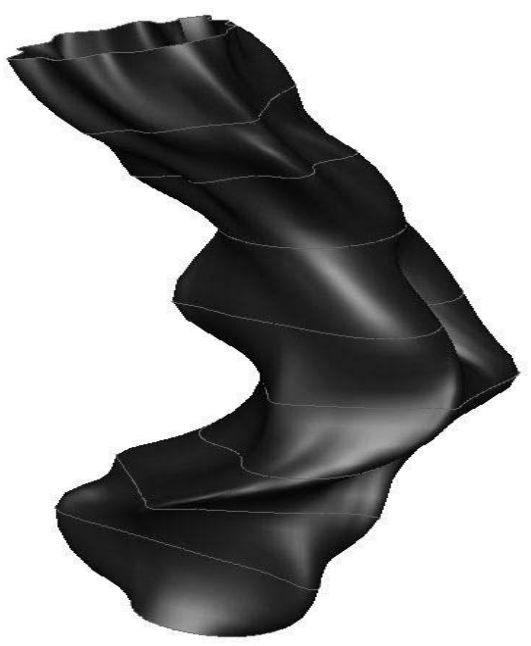

Fig. 8. Spine based shape manipulation for higher order PDE surfaces.

manipulation tool. The extension of the spine based shape manipulations techniques from Biharmonic case to higher order is relatively straightforward in that for higher order PDEs the polynomial is of higher degree and has greater flexibility.

There are several ways one can utilize the spine to manipulate the shape. One way of doing this is to imagine the vector constants where $\mathbf{a}_{00}+\mathbf{a}_{01}, \mathbf{a}_{02}, \ldots$, $\mathbf{a}_{2 N-1}$, as a set of design parameters whose values can be interactively changed. Another more intuitive method is to define the spine in terms of a spline control curve where the control points can be manipulated to change the shape of the spine and then $\mathbf{a}_{00}+\mathbf{a}_{01}, \mathbf{a}_{02}, \ldots, \mathbf{a}_{2 N-1}$ recomputed to change the shape of the surface. It is important to bear in mind that the constants $\mathbf{a}_{00}+\mathbf{a}_{01}, \mathbf{a}_{02}, \ldots, \mathbf{a}_{2 N-1}$ are 


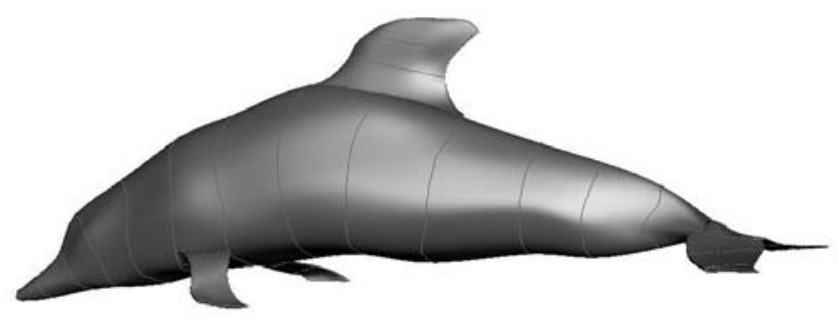

Fig. 9. Generic shape of a dolphin created interactively

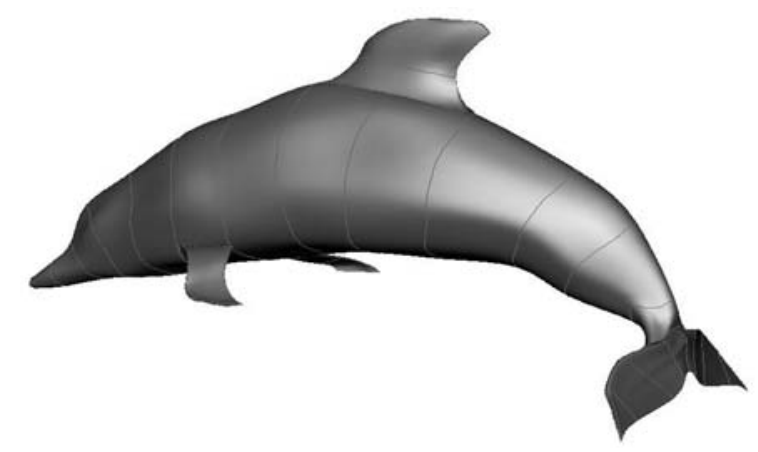

Fig. 10. Modified shape of the dolphin using boundary curves

dependent on the boundary conditions chosen. Therefore, when these parameters are interactively changed in order to manipulate the surface shape, the positions of the boundary curves will indeed be altered accordingly. Hence the user has a choice of using boundary conditions or the spine or even a combination of both to manipulate the shape.

Figure $7(\mathbf{a})$ shows the spine of corresponding to the higher order PDE surface patch shown in Figure (2). Figure (8) shows modified shape using the spine whereby treating it as a cubic spline curve and then manipulating its control points. The final shape of the spine corresponding to this modified geometry is shown in Figure $7(\mathbf{b})$.

\subsection{Examples of Interactive Design}

As one can clearly see that the above techniques of utilizing the boundary curves and using the spine on higher order PDE surfaces presents a powerful tool for complex shape design and manipulation interactively in real time. To demonstrate this further, here we present two examples of practical significance.

Figure (9) shows the generic shape of a dolphin created using 5 surface patches, i.e. a $14^{\text {th }}$ order patch for the main body of the dolphin, a $10^{\text {th }}$ order patch for the tail flukes and 3 Biharmonic patches for the lateral fins and the dorsal fin. In all 


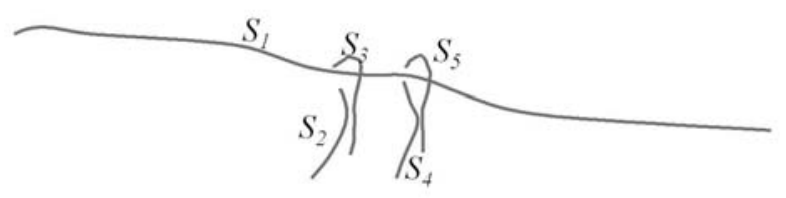

(a)

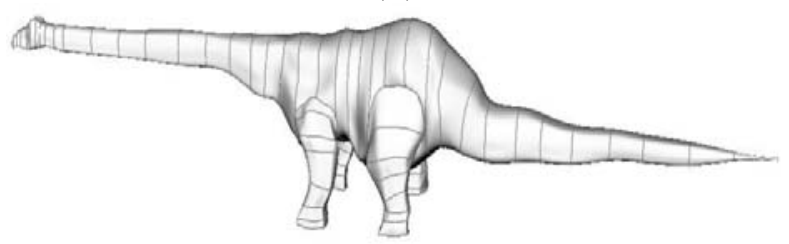

(b)

Fig. 11. A generic shape of Apatosaurus dinosaur created using 5 surface patches of higher order PDEs. (a) The spines corresponding to individual surface patches (b) The geometry of the dinosaur.

cases the boundary curves are taken to be position conditions thus enabling the curves to pass through the surface patches. The curves themselves are cubic splines which were created and manipulated within the interactive graphical environment. The curves which form the body attachment for the lateral fins and the dorsal fin respectively, lie on the main body surface where the portions have been trimmed off from the main body surface. These trimming processes were performed via the use of the $(u, v)$ parameter space using the techniques outlined $i^{38}$. Figure (10) shows an interactive modifications carried out to shape of the dolphin. These modification were carried by means of manipulating the four curves defining the back of the main body of the dolphin.

Figure (11)(b) shows the generic shape of an Apatosaurus dinosaur generated using 5 surface patches, i.e. a $40^{t h}$ order patch for the body (including the head, neck, main body and the tail) and four $8^{\text {th }}$ order patches for the legs. Again similar to the previous example of the dolphin, the curves forming the body attachments for the legs were taken on the main body surface where the portions have been trimmed off from the main body surface. Figure (11)(a) shows the spines (marked as $S_{1}, S_{2}, S_{3}, S_{4}$ and $S_{5}$ ) corresponding to the individual surface patches forming the dinosaur shape. Furthermore, for all the surface patches the corresponding boundary curves were taken to position conditions thus enabling the curves to pass through the surface patches. Again the curves themselves are cubic splines which were created and manipulated within the interactive graphical environment.

Figure (11)(a) shows the spine corresponding to the individual surface patches of the dinosaur geometry. Figure (12)(b) shows a modified geometry of the dinosaur using the spine corresponding to the body marked $S_{1}$. As described earlier the shape of the spine is manipulated by treating it as a cubic spline curve where the 


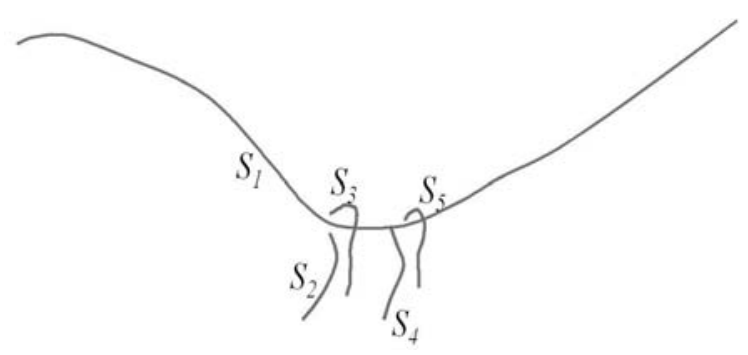

(a)

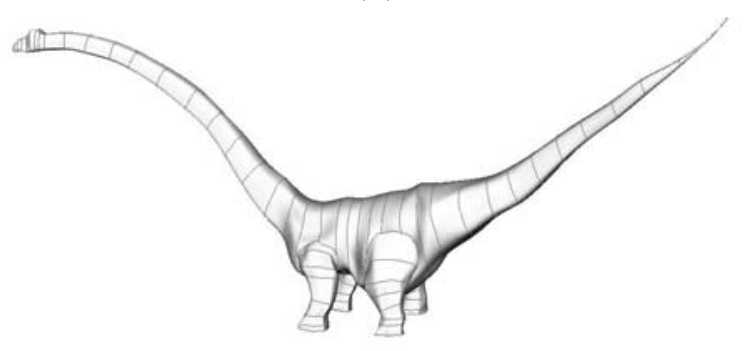

(b)

Fig. 12. Modified shape of the dinosaur using the spine corresponding to the body (a) The modified spine (b) Modified geometry of the dinosaur.

corresponding control points has been manipulated.

\subsection{Conclusions}

The discussions of this paper have been concerned with the generalization of the PDE based approach for surface generation whereby interactive design of complex geometry can be carried out. To this end the governing partial differential equation is generalized to arbitrary order where the equations are solved analytically. This solution scheme even in the case of general boundary conditions satisfies the boundary conditions exactly where the resulting surface has analytic representation allowing real time shape manipulation. Thus one could think that the usual Biharmonic equation design system is a subset of this generalized framework.

For the work described here the boundary conditions are defined as curves in 3 -space which can then be manipulated in real time. Furthermore, the spine of the corresponding higher order PDEs is also utilized as an interactive design tool for manipulating geometry. We have demonstrated the capacity of this method by means of design and manipulation of geometry corresponding to practical examples such as the dolphin and the dinosaur shape.

As one would observe for all the examples we have discussed in this paper we have used even number of boundary conditions. This is essential if we are to utilize the analytic solution form as presented in the paper. However, this is not necessarily 
a restriction on the type of geometry the method is capable of generating. In fact if we are presented with odd number of boundary conditions we could easily create a fictitious position boundary condition to make the number even and utilize analytic where, upon solving the even order equation, the undesired potion can be removed. Similarly the periodic nature of surfaces can also be removed by choosing to solve a portion of the surface along the $v$ parameter direction.

There are many possible extensions of this initial work. For example, we have presented a framework where the boundary conditions are taken to be curves most of which are cross sectional curves which lie on the surface. For interactive design purposes this is perhaps the most appropriate choice. Another possibility is to look into boundary conditions of mixed type, for which a different type of design metaphor will be required however.

For the purpose of real time design we have utilized analytic solutions to solve the generalized PDE where the boundary conditions must be of periodic nature. However, this is not a restriction on the method since one could utilize a numerical scheme to solve the arbitrary order PDE.

Much work has been done in the past using lower order PDEs on shape parameterization of generic designs where such parameterizations are ultimately utilized for automatic design optimizations. A shape parameterization based on higher order PDEs presents new avenues where it would be possible to parameterize complex geometry very efficiently and hence enabling the possibility of for automatic design optimization of very realistic design scenarios.

It is also noteworthy that the method discussed here has applications in other areas of computer graphics and computer-aided geometric design. For example, generation of higher order blend surfaces, geometric data interpolation as well smooth surface representations.

\section{Acknowledgements}

The author wishes to acknowledge the financial support received from the UK Engineering and Physical Sciences Research Council grants EP/C015118/01 and EP/D000017/01 through which this work was completed.

\section{References}

1. C. Bajaj and J. Blinn and J. Bloomenthal and M. Cani-Gascuel and A. Rockwood and B. Wyvill and G. Wyvill, Introduction to Implicit Surfaces (Morgan-Kaufmann, 1997).

2. M. I. G. Bloor and M. J. Wilson, Generating Blend Surfaces using Partial Differential Equations, Computer-Aided Design 21(3) (1989) 165-171.

3. M. I. G. Bloor and M. J. Wilson, Representing PDE Surfaces in terms of B-splines. Computer-Aided Design 22(6) (1990) 324-331.

4. M. I. G. Bloor and M. J. Wilson, Functionality in Solids Obtained from Partial Differential Equations. Computing, Computer-Aided Design 8 (1993) 21-42.

5. M. I. G. Bloor and M. J. Wilson, The Efficient Parameterization of Generic Aircraft Geometry, Journal of Aircraft 32(6) (1995) 1269-1275. 
6. M. I. G. Bloor and M. J. Wilson, Spectral Approximations to PDE Surfaces. Computer Aided Design, Computer-Aided Design 28(2) (1996) 145-152.

7. M. I. G. Bloor and M. J. Wilson, An Analytic Pseudo-Spectral Method to Generate Regular 4-sided PDE Surface Patch, Computer Aided Geometric Design 22(3) (2005) 203-219.

8. E. Catmull and J. Clark, Recursively Generated B-Spline Surfaces on Arbitrary Topological Meshes, Computer-Aided Design 10(6) (2002) 350-355.

9. C. W. Dekanski and M. I. G. Bloor and M. J. Wilson, The Representation of Marine Propeller Blades using the PDE Method, Journal of Ship Research 38(2) (1995) 108116.

10. M. Desbrun and M. Meyer and P. Schroder and A. H. Barr, Implicit Fairing of Irregular Meshes using Diffusion and Curvature Flow, Proceedings of SIGGRAPH 'g9 (1999) 317-324.

11. T. DeRose and M. Kass and T. Truong, Subdivision Surfaces in Character Animation, in Proceedings of SIGGRAPH 98, (Addison Wesley, 1998) pp. 85-94.

12. H. Du and H. Qin, Direct Manipulation and Interactive Sculpting of PDE surfaces, Computer Graphics Forum 19(3) (2000) 261-270.

13. H. Du and H. Qin, A Shape Design System using Volumetric Implicit PDEs, Computer Aided Design 36(11) (2004) 1011-1116.

14. G. Farin, Curves and Surfaces for Computer Aided Geometric Design, A Practical Guide, (Morgan Kaufmann, 2001).

15. E. Ferley and M. Cani and J. Gascuel, Practical Volumetric Sculpting, The Visual Computer 16(8) (2000) 469-480.

16. B. Fowler, Geometric Manipulation of Tensor Product Surfaces, Computer Graphics (1992 Symposium on Interactive 3D Graphics 25 (1992) 101-108.

17. C. F. Gerald and P. O. Wheatley Applied Numerical Analysis (Addison Wesley, 2003).

18. J. Hoschek and D. Lasser, Computer Aided Geometric Design, (A K Peters, 1993).

19. J. Huband and W. Li and R. Smith, An Explicit Representation of Bloor-Wilson PDE Surface Model by using Canonical Basis for Hermite Interpolation, Mathematical Engineering in Industry 7(4) (1999) 421-433.

20. J. Huband and W. Li, Extracting Design Parameters from Airplane Wing Data by using Bloor-Wilson PDE Surface Model, Mathematical Engineering in Industry 8 (2001) 239-252.

21. O. Kounchev, Multivariate Polysplines. Applications to Numerical and Wavelet Analysis (Academic Press, San Diego, 2001).

22. C. Loop, Smooth Subdivision Surfaces based on Triangles, Ph. D. Thesis, Department of mathematics, University of Utah, USA (1987).

23. T. W. Lowe and M. I. G. Bloor and M. J. Wilson, Integrating Physics-Based Modeling with PDE Solids for Geometric Design, in Functionality in blend design, Advanced in Design Automation, Vol 1, (ASME, New York, 1990) pp. 43-50.

24. J. Monterde and H. Ugail, On harmonic and biharmonic Bzier surfaces, Computing 21 (2004) 697-715.

25. M. E. Mortenson, Geometric Modelling (Wiley-Interscience, New York, 1985).

26. N. Paragios and R. Deriche, A PDE-based Level Set Approach for Detection and Tracking of Moving Objects, INRIA, France (1997).

27. L. Piegl and W. Tiller, The NURBS Book (Springer-Verlag, New York, 1997).

28. M. J. D. Powell, The Theory of Radial Basis Function Approximation in 1990, in Proceedings of 4th Summer School, Advances in numerical analysis. Vol. 2: Wavelets, subdivision algorithms, and radial basis functions, (Oxford University Press, Oxford, 1992) pp. 105-210. 
29. A. Rapporport and Y. Helor and M. Werman, Interactive Design of Smooth Objects with Probabilistic Point Constraints, ACM Transactions on Graphics 13 (1994) 156176.

30. A. Rappoport and S. Spitz, Interactive Boolean Operations for Conceptual Design of 3-D Solids, in Proceedings of the 24th annual conference on Computer graphics and interactive techniques, (ACM, New York, 1997) pp. 269-278.

31. A. Raviv and G. Elber, Three Dimensional Freeform Sculpting via Zero Sets of Scalar Trivariate Functions, in Proceedings of 5th ACM Symposium on Solid Modeling and Applications, (ACM, 1999) pp. 246-257.

32. L. Rudin and S. Osher and E. Fatemi, Nonlinear Total Variation Based Noise Removal Algorithms, JPhysica D 60(1-4) (1992) 259-268.

33. R. Schneider and L. Kobbelt, Generating Fair Meshes with G1 Boundary Conditions, in Proceedings of the Geometric Modeling and Processing 2000, (IEEE, Washington, 2000) pp. 251-261.

34. R. Szeliski and D. Terzopoulos, From Splines to Fractals, in Proceedings of the 16th annual conference on Computer graphics and interactive techniques, (ACM, New York, 1989) pp. 51-60.

35. D. Terzopoulos and H. Qin, Dynamic NURBS with Geometric Constraints for Interactive Sculpting, ACM Transactions on Graphics 13 (1994) 103-136.

36. W. Tiller, Rational B-splines for Curve and Surface Representation, IEEE Computer Graphics Applications 3 (1983) 61-69.

37. G. Turk and J. O'Brien, Modeling with Implicit Surfaces that Interpolate, ACM Transaction on Graphics 21(4) (2002) 855-873.

38. H. Ugail and M.I.G. Bloor and M.J. Wilson, Techniques for Interactive Design Using the PDE Method, ACM Transaction on Graphics 18(2) (1999) 195-212.

39. H. Ugail, Interactive Design and Optimisation of Thin-Walled Structures for Food Packaging, Journal of Optimisation and Engineering 4(4) (2003) 291-307.

40. H. Ugail and M.J. Wilson, Efficient Shape Parameterisation for Automatic Design Optimisation using a Partial Differential Equation Formulation, Computers and Structures 81(29) (2003) 2601-2609.

41. H. Ugail, On the Spine of a PDE Surface, in The Mathematics of Surface X, eds. R. R. Martin, and M. J. Wilson (Springer, Germany, 2003) pp. 366-376.

42. H. Ugail, Spine Based Shape Parameterisations for PDE Surfaces, Computing 72 (2004) 195-204.

43. L. A. Vese and T. F. Chan, A Multiphase Level Set Framework for Image Segmentation using the Mumford and Shah model, International Journal of Computer Vision 50(3) (2002) 271-293.

44. J. Warren and J. D. Warren and H. Weimer, Subdivision Methods for Geometric Design: A Constructive Approach (Morgan Kaufmann Publishers Inc,San Francisco, 2001).

45. A. Witkin and P. Heckbert, Using Particles to Sample and Control Implicit Surfaces, in Proceedings of SIGGRAPH 94, (ACM, 1994) pp. 269-277.

46. C. D. Woodward, Skinning Techniques for Interactive B-Spline Surface Interpolation, Computer Aided Design 20 (1988) 441-451.

47. L. You, P. Comninos, J.J. Zhang, PDE Blending Surfaces with C2 Continuity, Computers and Graphics 28(6) (2004), 895-906.

48. E. Zauderer, Partial Differential Equations of Applied Mathematics (WileyInterscience, New York, 1983).

49. J. J. Zhang and L. You, Fast Surface Modelling Using a 6th Order PDE, Computer Graphics Forum 23(3) (2004), 311-320. 